\title{
A Game Theoretical Framework for Dynamic Pricing-Based Routing in Self-Organized MANETs
}

\author{
Zhu Ji, Wei Yu and K. J. Ray Liu, Fellow, IEEE
}

\begin{abstract}
In self-organized mobile ad hoc networks (MANETs) where each user is its own authority, fully cooperative behaviors, such as unconditionally forwarding packets for each other or honestly revealing its private information, cannot be directly assumed. The pricing mechanism is one way to provide incentives for the users to act cooperatively by awarding some payment for cooperative behaviors. In this paper, we consider efficient routing in self-organized MANETs and model it as multi-stage dynamic pricing games. A game theoretical framework for dynamic pricing-based routing in MANETs is proposed to maximize the sender/receiver's payoff by considering the dynamic nature of MANETs. Meanwhile, the forwarding incentives of the relay nodes can also be maintained by optimally pricing their packet-forwarding services based on the auction rules and introducing the Cartel Maintenance enforcing mechanism. The simulation results illustrate that the proposed dynamic pricing-based routing approach has significant performance gains over the existing static pricing approaches.
\end{abstract} ing.

Index Terms - Ad Hoc Network, Pricing, Game Theory, Rout-

\section{INTRODUCTION}

$\mathbf{I}$ N RECENT years, mobile ad hoc networks (MANET) have received much attention due to their potential applications and the proliferation of mobile devices [1], [2]. In general, mobile ad hoc networks refer to wireless multi-hop networks formed by a set of mobile nodes without requiring centralized administration or fixed network infrastructure, in which nodes can communicate with other nodes out of their direct transmission ranges through cooperatively forwarding packets for each other. In traditional crisis or military situations, the nodes in a MANET usually belong to the same authority and work in a fully cooperative way of unconditionally forwarding packets for each other to achieve their common goals. Recently, the MANETs are also envisioned in civilian applications [3]-[9], where nodes typically do not belong to a single authority and may not pursue a common goal. Furthermore, such a network could be completely self-organizing, where the network would be run solely by the operation of the end-users. Consequently, fully cooperative behaviors cannot be directly assumed as the nodes are selfish to maximize their own interests. We refer to such networks as self-organized MANETs.

Manuscript received on August 1st, 2007 and revised on March 10th, 2008. This paper has been presented in part at IEEE INFOCOM'06, Barcelona, Spain, April 2006.

Zhu Ji is with Qualcomm, San Diego, CA 92121 USA (e-mail: zji@qualcomm.com).

Wei Yu is with Microsoft, Seattle, WA USA (e-mail: weiy@microsoft.com).

K. J. Ray Liu is with the Department of Electrical and Computer Engineering and Institute for Systems Research, University of Maryland, College Park, MD 20742 USA (e-mail: kjrliu@umd.edu).

Digital Object Identifier 10.1109/JSAC.2008.080917.
In order to analyze the selfish behaviors of network users for efficient self-organized wireless networking, game theoretical study is important to understanding and analyzing the interactions among intelligent users. Generally speaking, game theory [10], [11] models strategic interactions among agents using formalized incentive structures. It not only provides game models for efficient self-enforcing distributed design but also derives well-defined equilibrium criteria to measure the optimality of game outcomes for various scenarios. Considering self-organized MANETS, two types of game theoretical approaches have been proposed to analyze the interactions among network users and stimulate cooperation for self-organized networking: the reputation-based methods and pricing-based methods. In reputation-based methods such as [3]-[5], [7], [12], a node determines whether it should forward packets for other nodes or request other nodes to forward packets for it based on their past behaviors. In such schemes, by keeping monitoring packet forwarding activities, reputation effects and retribution are allowed to detect and isolate the misbehaving nodes from the rest of the network, thus enforcing the selfish nodes to behave cooperatively for better payoffs. In the pricing-based methods such as [6], [8], a selfish node will forward packets for other nodes only if it can get the properly-priced payment from those requesters as compensation. Although the above approaches have extensively studied the cooperation stimulation in self-organized MANETs for the packet forwarding among selfish users, cooperation stimulation for more advanced and sophisticated networking functionalities has not been fully addressed. One important fundamental issue needs to be further studied is how to carry out efficient self-organized routing in the dynamic scenarios of MANETs with selfish users.

Although the routing process is built upon successful packet forwarding among the nodes, the self-organized routing is much more complicated than packet forwarding for several reasons. First, the routing in ad hoc networks involves many selfish nodes at the same time for multi-hop packet forwarding and the behaviors of the selfish nodes may be correlated. Moreover, in MANETs, there usually exist multiple possible routes from the source to the destination. Furthermore, due to mobility, the available routes between the sources and the destinations may change frequently. In this paper, we refer to path diversity as the fact that in general there exist multiple routes between a pair of nodes, each with different characteristics, such as the number of hops, cost (or requested price), and valid time of this route. We refer to time diversity as the fact that due to the mobility, dynamic topology, and traffic variations, the routes between two nodes will keep 
changing over time. In order to achieve efficient routing in self-organized MANETs, a comprehensive study needs to be carried out considering the above aspects.

Several approaches have been proposed to exploit the path diversity during the routing process in self-organized MANETs such as [13]-[15]. Based on the ideas of the auctionlike pricing and routing protocols for the Internet [16], [17], the authors in [13]-[15] have introduced some auction-like methods for the cost-efficient and truthful routing in MANETs, where the sender-centric Vickrey auction has been adopted to discover the most cost-efficient routes, which has the advantage that its incentive compatible property ensures the truthful routing among the nodes. Router-based auction approaches [18], [19] have also been proposed to encourage the packet-forwarding in MANETs, where each router constitutes an auction market instead of submitting bids to the sender. Besides, a strategy-proof pricing algorithm for the truthful multi-cast routing has been proposed in [20].

Although the existing pricing-based approaches [13]-[15] have achieved some success in cost-efficient and incentivecompatible routing for MANETs with selfish nodes, most of them assume that the network topology is fixed or the routes between the sources and the destinations are known and pre-determined. Further, none of the existing approaches have addressed how to exploit the time diversity for efficient routing. In order to have optimal pricing-based routing, both path diversity and time diversity of MANETs should be exploited. Specifically, the source (here we assume the source pays to the forwarding nodes) is responsible for exploiting the path diversity, such as introducing competition among the multiple available routes through auction, to minimize the payment needed at the current stage. Each node also needs to exploit the time diversity to maximize its overall payoff over time. In each stage the source adaptively decides the number of packets being transmitted according to the price it needs to pay, which is determined by the current routing conditions. For instance, when the routing conditions are good (i.e., the cost to transmit a packet is low), more packets should be transmitted in the current stage; otherwise, less or no packets should be transmitted in the current stage.

In this paper, we consider the routing process as multi-stage dynamic games and propose an optimal pricing-based approach to dynamically maximize the sender/receiver's payoff over multiple routing stages considering the dynamic nature of MANETs, meanwhile, keeping the forwarding incentives of the relay nodes by optimally pricing their packet-forwarding actions based on the auction rules. The main contribution of this paper are multi-fold: First, by modeling the pricingbased routing as a dynamic game, the senders are able to exploit the time diversity in MANETs to increase their payoffs by adaptively allocating the packets to be transmitted into different stages. Considering the mobility of the nodes, the possible routes for each source-destination pair are changing dynamically over time. According to the path diversity, the sender will pay a lower price for transmitting packets when there are more potential routes. Thus, the criterion for allocation can be developed based on the fact that the sender prefers to send more packets in the stage with lower costs. The Cartel Maintenance mechanism is introduced to ensure the cooperation within each route. Second, an optimal dynamic programming approach is proposed to implement efficient multi-stage pricing for self-organized MANETs. Specifically, the Bellman equation is used to formulate and analyze the above dynamic programming problem by considering the optimization goal in terms of two parts: current payoffs and future opportunity payoffs. A simple allocation algorithm is developed and its optimality is proved based on the auction structure and routing dynamics. Third, the path diversity of MANETs is exploited using the optimal auction mechanism in each stage. The application of the optimal auction [21] makes it possible to separately study the optimal allocation problem and the mechanism design of the auction protocol based on the well-known Revenue Equivalence Theorem [21], which simplifies the dynamic algorithm while keeping the optimality.

The reminder of this paper is organized as follows: The system model of self-organized MANETs are illustrated in Section 2. In Section 3, we formulate the pricing process as dynamic games based on the system model. In Section 4, the optimal dynamic auction framework is proposed for the optimal pricing and allocation of the multi-stage packet transmission. In Section 5, extensive simulations are conducted to study the performance of the proposed approach. Finally, conclusions are drawn in Section 6.

\section{SySTEM MOdeL}

In this paper we consider self-organized mobile ad hoc networks where nodes belong to different authorities and have different goals. We assume that each node is equipped with a battery with limited power supply, can freely move inside a certain area, and communicates with other nodes through wireless connections. For each node, packets are scheduled to be generated and delivered to certain destinations with each packet having a specific delay constraint, that is, if a packet cannot reach the destination within its delay constraint, it will become useless.

In our system model, we assume all nodes are selfish and rational, that is, their objectives are to maximize their own payoff, not to cause damage to other nodes. However, node are allowed to cheat whenever they believe cheating behaviors can help them increasing their payoff. Since nodes are selfish and forwarding packets on behalf of others will incur some cost, without necessary compensation, nodes have no incentive to forward packets for others. In our system model, we assume that if a packet can be successfully delivered to its destination, then the source and/or the destination of the packet can get some benefits, and when a node forwards packets for others, it will ask for some compensation, such as virtual money or credits [6], from the requesters to at least cover its cost. In our system model, to simplify our illustration, we assume that the source of a packet pays to the intermediate nodes who have forwarded the packet for it. However, the proposed schemes can also be easily extended to handle the situation that the destinations pay. Like in [6], we assume that there exist some bank-like centralized management points, whose only function is to handle the billing information, such as performing credit transfer among nodes based on the submitted information by these nodes. Each node only needs to contact these central banking points periodically or aperiodically. 


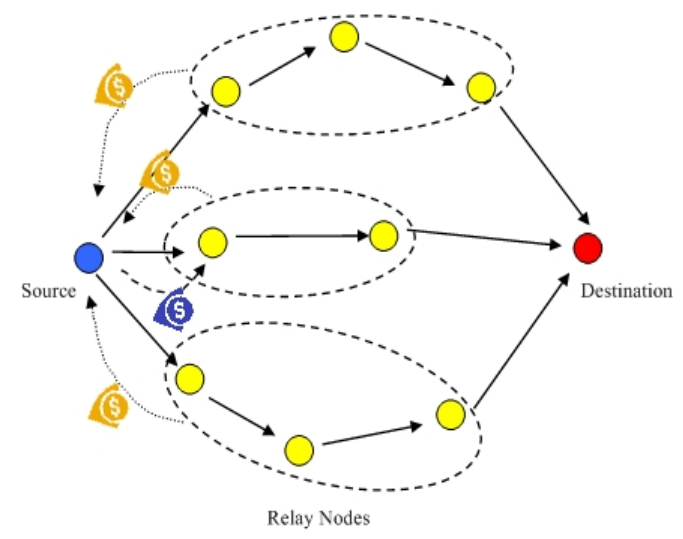

Fig. 1: Pricing-based routing in self-organized MANETs.

In general, due to the multi-hop nature of ad hoc networks, when a node wants to send a packet to a certain destination, a sequence of nodes need to be requested to help forwarding this packet. We refer to the sequence of (ordered) nodes as a route, the intermediate nodes on a route as relay nodes, and the procedure to discover a route as route discovery. The routing protocols are important for MANETs to establish communication sessions between each source-destination pair. Here, we consider the on-demand (or reactive) routing protocols for ad hoc networks, in which a node attempts to establish a route to some destination only when it needs to send packets to that destination. Since on-demand routing protocols are able to handle many changes of node connectivity due to the node's mobility, they perform better than periodic (or proactive) routing protocols in many situations [22]-[24] by having much lower overheads. In MANETs, due to the mobility, nodes need to frequently perform route discovery. In this paper, we refer to the interval between two consecutive route discovery procedures as a routing stage, and assume that for each source-destination pair, the selected route between them will keep unchanged in the same routing stage. Furthermore, to simplify our analysis, we assume that for each sourcedestination pair, the discovered routes in different routing stages are independent.

After performing route discovery in each stage, multiple forwarding routes can be exploited between the source and the destination. Assume there are $\ell$ possible routes and let $v_{i, j}$ be the forwarding cost of the $j$ th node on the $i$ th route, which is also referred to as the node type in this paper. Considering possible node mobility in MANET, $\ell$ and $v_{i, j}$ are no longer fixed values, which can be modelled as random variables. Let the probability mass function (PMF) of $\ell$ be $\tilde{f}(\ell)$ and the corresponding cumulative density function $(\mathrm{CMF})$ be $\tilde{F}(\ell)$. And, $v_{i, j}$ is characterized by its probability density function (PDF) $\hat{f}_{i, j}$ and the cumulative density function $(\mathrm{CDF}) \hat{F}_{i, j}$. Define the cost vector of the $i$ th route as $\mathbf{v}_{i}=\left\{v_{i, 1}, v_{i, 2}, \ldots, v_{i, h_{i}}\right\}$, where $h_{i}$ is the number of forwarding nodes on the $i$ th route. Thus, we have the total cost on the $i$ th route $r_{i}=\sum_{j=1}^{h_{i}} v_{i, j}$,

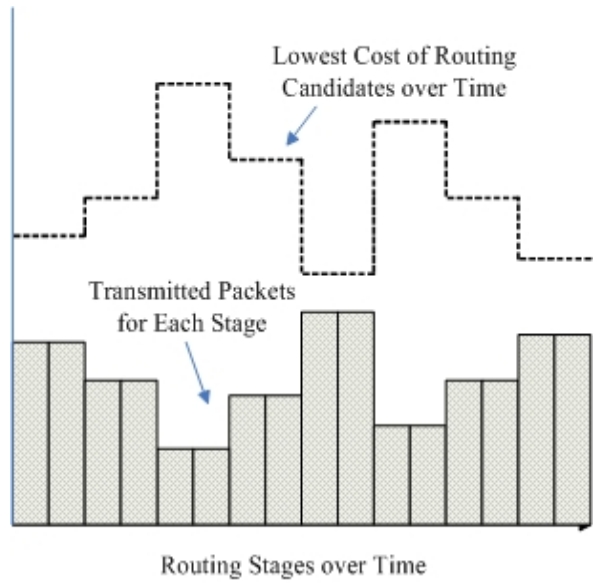

Fig. 2: Dynamic pricing-based routing considering time diversity.

which is also a random variable. Let the PDF and CDF of $r_{i}$ be $f_{i}$ and $F_{i}$, respectively.

Figure 1 illustrates our system model by showing a network snapshot of pricing-based multi-hop routing between a sourcedestination pair. It can be seen from this figure that there are three routing candidates with different number of hops and routing costs (such as energy-related forwarding costs) between the source-destination pair. Each route will bid as one entity for providing the packet forwarding service for the source-destination pair at this routing stage. Then, the source will choose the route with the lowest bid to transmit the packets. The price that the source pays to the selected route may be equivalent to the asked price or include a premium than the true forwarding cost. Note that the asking prices from each route and the payment from the source may vary according to the applied pricing mechanisms. Further, the payment that the source provides to the selected route needs to be shared among the nodes on the selected route in a way that no node on the selected route has incentive to deviate from the equilibrium strategy. Considering the network dynamics due to the node mobility, dynamic topology or channel fading, the number of available routes, the number of required hops and the forwarding costs will change over time. In Figure 2, we consider a dynamic scenario and illustrate the relationship of the number of packets to be transmitted and the lowest cost of the available routes at each stage. In order to maximize its payoff by utilizing the time diversity, the source tends to transmit more packets when the cost is lower and transmit less packets when the cost is higher. The optimal relationship between them will be derived in later sections.

\section{Pricing Game Models}

In this paper, we model the process of establishing a route between a source and a destination node as a game. The players of the game are the network nodes. With respect to a given communication session, any node can play only one of the following roles: sender, relay node, or destination. In selforganized MANET, each node's objective is to maximize its 
own benefits. Specifically, from the sender's point of view, he/she aims to transmit its packets with the least possible payments; from the relaying nodes' points of view, they want to earn the payment which not only covers their forwarding cost but also gain as much extra payment as possible; while from the network designers' point of view, they prefer that the network throughput and/or lifetime can be maximized. Therefore, the source-destination pair and the nodes on the possible forwarding routes construct a non-cooperative pricing game [10]. Since the selfish nodes belong to different authorities, the nodes only have the information about themselves and will not reveal their own types to others unless efficient mechanisms have been applied to guarantee that truth-telling does not harm their interests. Generally, such non-cooperation game with imperfect information is complex and difficult to study as the players do not know the perfect strategy profile of others. But based on our game setting, the well-developed auction theory can be applied to analyze and formulate the pricing game.

The auction games belong to a special class of game with incomplete information known as games of mechanism design, in which there is a "principal" who would like to condition his actions on some information that is privately known by the other players, called "agents". In auction, according to an explicit set of rules, the principle (auctioneer) determines resource allocation and prices on the basis of bids from the agents (bidders). In the pricing game, the source can be viewed as the principle, who attempts to buy the forwarding services from the candidates of the forwarding routes. The possible forwarding routes are the bidders who compete with each other for serving the source node, by which they may gain extra payments for future use. In order to maximize their own interests, the selfish forwarding nodes will not reveal their private information, i.e., the actual forwarding costs, to others. They compete for the forwarding request by eliciting their willingness of the payments in the forms of bids. Thus, because of the path diversity of MANET, the sender is able to lower its forwarding payment by the competition among the routing candidates based on the auction rules. It is important to note that instead of considering each node as a bidder [13], [15], we consider each route as a bidder in this paper, which has the following advantages: First, by considering the nodes on the same forwarding route as one entity, the sender can fully exploit the path diversity to maximize its own payoffs by lowering the bidding premium for ensuring the truth-telling for each node [13] on the route. Second, since it has been proved in [15] that there does not exist a forwardingdominant protocol for ad hoc pricing games, we analyze the pricing-based routing in a two-step approach: first study the payoff-maximization allocation by considering the route-based bidding, and then derive the truth-telling profit-sharing among the nodes on the selected route based on repeated game theory. Moreover, less bidding information is required for route-based approach compared to [13] as each route is considered as only one bidding entity.

In this section, we first consider the static pricing game (SPG), which is only played once for the fixed topology. Then, the dynamic pricing game (DPG) is studied and formulated considering playing the pricing game for multiple stages.

\section{A. The Static Pricing Game}

In this subsection, we study the static pricing game model. By taking advantage of the auction approach, our goal is to maximize the profits of the source-destination communication pair for transmitting packets while keeping the forwarding incentives of the forwarding routes. Specifically, considering an auction mechanism $(\mathbf{Q}, \mathbf{M})$ consists of a pair of functions $\mathbf{Q}: \mathcal{D} \rightarrow \mathcal{P}$ and $\mathbf{M}: \mathcal{D} \rightarrow R^{N}$, where $\mathcal{D}$ is the set of announced bids, $\mathcal{P}$ is the set of probability distributions over the set of routes $\mathcal{L}$. Note that $Q_{i}(\mathbf{d})$ is the probability that the $i$ th route candidate will be selected for forwarding and $M_{i}(\mathbf{d})$ is the expected payment for the $i$ th route, where $\mathbf{d}$ is the vector of bidding strategies for all routes, i.e., $\mathbf{d}=\left\{d_{1}, d_{2}, . ., d_{\ell}\right\} \in \mathcal{D}$. Then, the payoff function of the $i$ th forwarding route can be represented as follows

$$
U_{i}\left(d_{i}, d_{-i}\right)=M_{i}\left(d_{i}, d_{-i}\right)-Q_{i}\left(d_{i}, d_{-i}\right) \cdot r_{i} .
$$

Before studying the equilibria of the auction game, we first define the direct revelation mechanism as the mechanism in which each route bids its true cost, $d_{i}=r_{i}$. The Revelation Principle [21] states that given any feasible auction mechanism, there exists an equivalent feasible direct revelation mechanism which gives to the auctioneer and all bidders the same expected payoffs as in the given mechanism. Thus, we can replace the bids $\mathbf{d}$ by the cost vector of the routes, i.e., $\mathbf{r}=\left\{r_{1}, r_{2}, \ldots, r_{L}\right\}$ without changing the outcome and the allocation rule of the auction game. Therefore, the equilibrium of the SPG can be obtained by solving the following optimization problem to maximize the sender's payoff while providing incentives for the forwarding routes

$$
\begin{gathered}
E_{\ell, \mathbf{r}}\left[\max _{\mathbf{Q}, \mathbf{M}}\left\{g \cdot \sum_{i=1}^{\ell} Q_{i}(\mathbf{r})-\sum_{i=1}^{\ell} M_{i}(\mathbf{r})\right\}\right] \\
\text { s.t. } \quad U_{i}\left(r_{i}, d_{-i}\right) \geq U_{i}\left(d_{i}, d_{-i}\right), \forall d_{i} \in \mathcal{D} \\
Q_{i}(\mathbf{r}) \in\{0,1\}, \sum_{i=1}^{\ell} Q_{i}(\mathbf{r}) \leq 1 .
\end{gathered}
$$

where the constraint (3) is also referred as the incentive compatibility (IC) constraint, which ensures the users to report their true types, and $g$ is the marginal profit of transmitting one packet. Note that $\{0,1\}$ represents a set having two elements, 0 and 1.

\section{B. The Dynamic Pricing Game}

Considering the dynamic nature of MANET, the network topology may change over time due to the mobility of the nodes. Thus, the route discovery needs to be performed frequently. Moreover, for different routing stages, there may exist different number of available routes with different number of hops. It is important for each source-destination pair to decide the transmission and payment behaviors for each stage according to the route conditions. Therefore, the pricing game under such dynamic situation can no longer be modelled as static games. Game theorists use the concept of dynamic games to model such multi-stage games and analyze the longrun behaviors of players. In dynamic games, the strategies of the players not only depend on the opponents' current 
strategies but also the past outcomes of the game and the future possible actions of other players. Our pricing game for MANET falls exactly into the category of dynamic games. In this paper, we will focus on studying the dynamic pricing game.

Intuitively, the sender prefers to transmit more packets when more routing candidates are available and the number of hops is small. Because, considering the application of auction protocols in each stage, the sender has a higher probability to get the service with a lower price when there are more bidders (routes) with lower type values. Moreover, the practical constraints in MANET need to be considered in DPG, such as the delay constraint of packet transmission or the bandwidth constraint of the maximal number of packets being able to be transmitted within an unit time duration. Therefore, in order to maximize their profits, the sourcedestination pair needs not only to optimally allocate the packets to the routes within one time period but also to schedule the packets for all periods. In our DPG, it is important to note that the optimal packet transmission strategy for each source-destination pair is affected by both the past plays and the future possible outcomes. Generally speaking, the packet transmission decision is made by comparing the current transmission profit and future opportunity profits. Also, due to the delay and bandwidth constraints, the past transmission plays affect current decision-making. Capturing the dynamics becomes the key to the optimal solution of our DPG. Let $\ell_{t}$ denote any realization of the route number at the $t$ th stage and $\mathbf{r}_{t}$ be a realization of the types of all routing candidates at the $t$ th stage. The probabilistic structures of $\ell_{t}$ and $\mathbf{r}_{t}$ are different for different ad hoc networking scenarios. By properly choosing the stage time-duration, the dependency of routing dynamics across stages can be negligible. Consider a $T$-period dynamic game, the overall payoff maximization problem for the source-destination pair can be formulated as follows.

$$
\begin{gathered}
\sum_{t=1}^{T} \beta^{t} \cdot E_{\ell_{t}, \mathbf{r}_{t}}\left[\max _{\mathbf{Q}, k_{t}}\left\{\left[G\left(\mathcal{K}_{t}\right) \cdot \sum_{i=1}^{\ell_{t}} Q_{i}-k_{t} \cdot \sum_{i=1}^{\ell_{t}} M_{i}\left(\mathbf{r}_{t}\right)\right]\right\}\right] \\
\text { s.t. } \quad U_{i, t}\left(r_{i, t}, d_{-i, t}\right) \geq U_{i, t}\left(d_{i, t}, d_{-i, t}\right), \forall d_{i, t} \in \mathcal{D} \\
Q_{i} \in\{0,1\}, \sum_{i=1}^{L} Q_{i} \leq 1 . \\
k_{t} \leq \mathcal{B}, \sum_{t=1}^{T} k_{t}=M .
\end{gathered}
$$

where $k_{t}$ is the number of packets transmitted in the $t$ th stage and $\mathcal{K}_{t}$ is the vector of the numbers of the transmitted packets in the first $T-t+1$ stages, which can be represented as $\mathcal{K}_{t}=\left\{k_{T}, k_{T-1}, \ldots, k_{t}\right\}$. Note that a smaller $t$ in this paper stands for a later time stage. Here, $G\left(\mathcal{K}_{t}\right)$ is the profit that the sender gains in the $t$ th stage, which may not only depend on how many packets are transmitted in current stage, i.e., $k_{t}$, but also be affected by how many packets have been transmitted in previous stages, $\mathcal{K}_{t+1}$. Considering the rate-distortion theory [25], we assume the profit function is concave in $k_{t}$. For example, the marginal profit of transmitting one more packet when a lot of packets have already been transmitted should be limited. Also, the subscript $t$ indicates the $t$ th routing stage and $\beta$ is the discount factor for multistage games. Considering different applications, $\beta$ needs to be determined differently: for real-time applications such as video streaming or voice, $\beta$ is a smaller value less than 1 so that the payoff at current stage contributes most to the overall payoff; for non-realtime applications such as data transmission, $\beta$ can be chosen to be very close to 1 so that the overall payoff is almost evenly affected by the payoff at each stage. Note that $T$ and $\mathcal{B}$ are the delay constraint and the bandwidth constraint, respectively. $M$ is the total number of packets to be transmitted within $T$ stages.

The above DPG formulation (4) extends the optimal pricing problem to the time dimension, which can exploit the potential of time diversity in the self-organized ad hoc network considering its dynamic nature. For example, if current routing condition is not good, the user could hold its transmission for the future, during which the routing cost may become lower. Thus, seemingly current payoff loss can be compensated by much higher future payoff so that the overall payoff can be optimized.

It is worth mentioning that directly solving the nonlinear integer programming problem is very difficult. Because, not only does the current routing realization affect the allocation decision, but also the past play and allocation decision influence the feasible actions and payoff functions in the current period.

\section{Optimal Dynamic Pricing-Based Routing}

In order to achieve efficient self-organized routing in the DPG considering the dynamic nature of MANETs, we propose the optimal pricing-based routing approach in this section. First, the optimal auction mechanism is considered for maximizing the payoffs for the source-destination pair while keeping the forwarding incentives of the relaying nodes. Then, the dynamic multi-stage game is further formulated using the optimal auction and dynamic programming approach. Finally, the mechanism design and the profit-sharing among the nodes on the selected route are considered for the proposed approach.

\section{A. The Optimal Auction for Static Pricing-Based Routing}

In Section 2, we have formulated the static pricing game based on the auction principles as the optimization problem (2). Here, we further utilize the results of the optimal auction [26] to simplify the optimization problem. From [26], we know that by considering the optimal auction, the sender's expected total payoff can be expressed only in terms of the allocation $\mathbf{Q}$, which is independent of the payment to each route candidate. Specifically, the optimization problem (2) can be rewritten as follows.

$$
\begin{gathered}
E_{\ell, \mathbf{r}}\left[\max _{\mathbf{Q}}\left\{g \cdot \sum_{i=1}^{\ell} Q_{i}(\mathbf{r})-\sum_{i=1}^{\ell} J_{i}\left(r_{i}\right) Q_{i}(\mathbf{r})\right\}\right], \\
\text { s. t. } \quad Q_{i}(\mathbf{r}) \in\{0,1\}, \quad \sum_{i=1}^{\ell} Q_{i}(\mathbf{r}) \leq 1 .
\end{gathered}
$$


where $J\left(r_{i}\right)=r_{i}+1 / \rho\left(r_{i}\right)$, and $\rho\left(r_{i}\right)=f_{i}\left(r_{i}\right) / F_{i}\left(r_{i}\right)$ is the hazard rate [26] function associated with the distribution of the routing cost. Note that $J\left(r_{i}\right)$ is also called the virtual type of the $i$ th player. It's proved in [26] that the solution of the above optimization also satisfies the incentive compatible constraint. The assumptions for the above formulation are rather general: (1) $F$ is continuous and strictly increasing, (2) the allocations $Q_{i}\left(r_{i}, r_{-i}\right)$ are increasing in $r_{i}$. From (5) and the Revenue Equivalence Theorem, it follows that all mechanisms that result in the same allocations $\mathbf{Q}$ for each realization of $\mathbf{r}$ yield the same expected payoff. Thus, in order to obtain the optimal pricing strategies, the mechanism design process proceeds in two steps: First, find the optimal allocation $\mathbf{Q}(\mathbf{r})$; second, find an implementable mechanism that produces $\mathbf{Q}$ for each realization $\mathbf{r}$. By using the optimal auction approach for pricing, the payoff-maximized allocation for the sender is to choose the route with the minimal virtual type $J\left(r_{i}\right)$ when $g-J\left(r_{i}\right) \geq 0$, otherwise the sender will not transmit the packet as it will cause negative payoff and violate his individual rationality. Therefore, if we assume $J(v)$ is strictly increasing in $v$, we can define $v *=\max _{v}\{(g-J(v))=0\}$ as the reserved price for the sender, which is the largest payment he/she can offer for transmitting a packet. Note that the distributions that have increasing $J(v)$ include the uniform, normal, logistic, exponential distributions, etc.

Based on the above discussion, we find that the static pricing game is not efficient if the current routing realization shows a high cost. Considering the dynamic properties of MANET, a more efficient pricing mechanism can be achieved by studying it as a multistage game and optimally allocating the packet transmissions over multiple time periods.

\section{B. The Optimal Dynamic Auction for Dynamic Pricing-Based Routing}

Considering the optimal auction results in the DPG model formulated in Section 2, we further propose the optimal dynamic auction framework for pricing in self-organized MANET. As it is difficult to directly solve (4), we study the dynamic programming approach in our proposed framework to simplify the multistage optimization problem.

Define the value function $V_{t}(x)$ as the maximum expected profit obtainable from periods $t, t-1, \ldots, 1$ given that there are $x$ packets to be transmitted within the constraint of time periods. Simplifying (4) using the Bellman equation, we have the maximal expected profit $V_{t}(x)$ written as follows

$$
\begin{array}{r}
V_{t}(x)=E_{\ell_{t}, \mathbf{r}}\left[\operatorname { m a x } _ { \mathbf { Q } , k _ { t } } \left\{\left[G\left(\mathcal{K}_{t}\right) \cdot \sum_{i=1}^{\ell_{t}} Q_{i}-k_{t} \cdot \sum_{i=1}^{\ell_{t}} J\left(v_{i}\right) Q_{i}\right]\right.\right. \\
\left.\left.+\beta \cdot V_{t-1}\left(x-k_{t}\right)\right\}\right],
\end{array}
$$

$$
\text { s.t. } \quad Q_{i}(\mathbf{r}) \in\{0,1\}, \quad \sum_{i=1}^{\ell_{t}} Q_{i}(\mathbf{r})=1, \quad k_{t} \leq \mathcal{B} .
$$

Moreover, the boundary conditions for the above dynamic programming problem are

$$
V_{0}(x)=0, x=1, \ldots, M,
$$

Recall that we have the delay constraint $T$ of the maximal allowed time stages and the bandwidth constraint $\mathcal{B}$ of the maximal number of packets able to be transmitted for each stage. Based on the principle of optimality in [27], an allocation $\mathbf{Q}$ that achieves the maximum in (6) given $x, t$ and $\mathbf{r}$ is also the optimal solution for the overall optimization problem (4). Note that the above formulation is similar to that of the multi-unit sequential auction [28] studied by the economists.

First, note that from (6) and the monotonicity of $J(\cdot)$, it is clear that if the sender transmits $k$ packets within one time period, these packets should be all awarded to the forwarding route with the lowest cost $r_{i}$. Therefore, define

$$
\begin{array}{r}
R_{t}(k)=\max _{\mathbf{Q}}\left\{G\left(\mathcal{K}_{t}\right) \cdot \sum_{i=1}^{\ell_{t}} Q_{i}(\mathbf{r})-k \cdot \sum_{i=1}^{\ell_{t}} J\left(r_{i}\right) Q_{i}(\mathbf{r}):\right. \\
\left.Q_{i}(\mathbf{r}) \in\{0,1\}, \sum_{i} Q_{i}(\mathbf{r})=1\right\},
\end{array}
$$

which can also be solved and written as

$$
R_{t}(k)= \begin{cases}0 & \text { if } k=0, \\ G\left(k, \mathcal{K}_{t+1}\right)-k \cdot J\left(r_{(1)}\right) & \text { if } k>0,\end{cases}
$$

where $r_{(1)}$ means the lowest cost of the forwarding routes. Thus, the dynamic optimization objective (6) can therefore be rewritten in terms of $R_{t}(k)$ as follows:

$V_{t}(x)=E_{\ell_{t}, \mathbf{r}}\left[\max _{0 \leq k_{t} \leq \min \{\mathcal{B}, x\}}\left\{R_{t}\left(k_{t}\right)+\beta \cdot V_{t-1}\left(x-k_{t}\right)\right\}\right]$,

which is also subject to (7). Let $k_{t}^{*}(x)$ denote the optimal solution above, which is the optimal number of packets to be transmitted on the winning route at the $t$ th stage given remaining capacity $x$. Letting $\triangle R_{t}(i) \equiv R_{t}(i)-R_{t}(i-1)$ and $\triangle V_{t}(i) \equiv V_{t}(i)-V_{t}(i-1)$, we can rewrite the maximal expected profit $V_{t}(x)$ as

$$
\begin{aligned}
& V_{t}(x)=E_{\ell_{t}, \mathbf{r}}\left[\operatorname { m a x } _ { 0 \leq k _ { t } \leq \operatorname { m i n } \{ \mathcal { B } , x \} } \left\{\sum _ { i = 1 } ^ { k _ { t } } \left[\triangle R_{t}(i)\right.\right.\right. \\
& \left.\left.\left.-\beta \cdot \triangle V_{t-1}(x-i+1)\right]\right\}\right]+\beta \cdot V_{t-1}(x) .
\end{aligned}
$$

The above formulation will help us to simplify the optimal dynamic pricing problem.

Then, in order to solve the dynamic pricing problem (6)(7), we need to first introduce the following lemmas based on (11).

Lemma 1: If $\triangle V_{t-1}(x) \geq \triangle V_{t-1}(x+1)$, then $k_{t}^{*}(x) \leq$ $k_{t}^{*}(x+1) \leq k_{t}^{*}(x)+1, \forall x \geq 0$.

Proof: We study the left hand side (LHS) inequality first. If $k_{t}^{*}(x)=0$, the inequality holds true. If $k_{t}^{*}(x)>0$ (6) and considering the assumption $\triangle V_{t-1}(x) \geq \triangle V_{t-1}(x+1)$, the optimal allocation $k_{t}^{*}(x+1)$ may be higher due to the additional packet in queue. Hence, $k_{t}^{*}(x+1) \geq k_{t}^{*}(x)$.

As for the right hand side (RHS) inequality, we prove it by contradiction. Assume $k_{t}^{*}(x+1) \geq k_{t}^{*}(x)+2$. From (9), we know that $R(k)$ is decreasing in its argument. Further, from (11) and the assumption of this lemma $\triangle V_{t-1}(x) \geq$ $\triangle V_{t-1}(x+1)$, we obtain that achieving the optimal $k$ for the $t$ th stage in (11) is equivalent to finding the maximal $k$ 
satisfying the following inequality

$$
\triangle R_{t}(k)>\beta \cdot \triangle V_{t-1}(x-k+1) .
$$

Therefore, given the optimal $k_{t}^{*}(x+1)$, we have

$\triangle R_{t}(m)>\beta \cdot \triangle V_{t-1}(x+1-m+1)$, for $m=1,2, \ldots, k_{t}^{*}(x+1)$.

As we assume $k_{t}^{*}(x+1) \geq k_{t}^{*}(x)+2$ and letting $m=k_{t}^{*}(x)+2$ in (13), we obtain

$$
\begin{aligned}
\triangle R_{t}\left(k_{t}^{*}(x)+2\right) & >\beta \cdot \Delta V_{t-1}\left(x+1-\left(k_{t}^{*}(x)+2\right)+1\right) \\
& =\beta \cdot \Delta V_{t-1}\left(x-\left(k_{t}^{*}(x)+1\right)+1\right) \cdot(14)
\end{aligned}
$$

Since $R(k)$ is decreasing in $k,(14)$ can be further written as

$$
\begin{aligned}
\triangle R_{t}\left(k_{t}^{*}(x)+1\right) & \geq \triangle R_{t}\left(k_{t}^{*}(x)+2\right) \\
& >\beta \cdot \Delta V_{t-1}\left(x-\left(k_{t}^{*}(x)+1\right)+1015\right)
\end{aligned}
$$

Considering the optimality criterion of $k_{t}^{*}(x)$ in (12), $k_{t}^{*}(x)$ should be the largest number of packets satisfying (12). Therefore, (15) contradicts the optimality of $k_{t}^{*}(x)$. The RHS inequality is proved.

It can be seen from the proof of Lemma 1 that the optimal allocation of packet transmission over multiple stages can also be determined under the condition $\triangle V_{t-1}(x) \geq \triangle V_{t-1}(x+1)$. Then, we will prove the above condition holds for all $t$ in the following lemma.

Lemma 2: $\triangle V_{t}(x)$ is decreasing in $x$ for any fixed $t$ and is increasing in $t$ for any fixed $x$.

Proof: See the Appendix.

The idea of this lemma can also be illustrated in an intuitive way as follows. At any fixed time period, the marginal benefit $\triangle V_{t}(x)$ of each additional packet declines because the future possible routes are limited; therefore, the chance of transmitting the additional packet at low prices also decreases. Similarly, for any given remaining packet number $x$, the marginal benefit of an additional packet increases with $t$, because more number of possible future routes are available when more remaining time periods; therefore, the chance of getting a higher marginal benefit goes up. Also, Lemma 2 relaxes the assumption of Lemma 1 and we always have $k_{t}^{*}(x) \leq k_{t}^{*}(x+1) \leq k_{t}^{*}(x)+1, \forall x \geq 0$.

Considering Lemma 1 and Lemma 2, the optimal allocation of packet transmission for the proposed dynamic auction framework can be characterized by the following theorem.

Theorem 1: For any realization $\left(\ell_{t}, \mathbf{r}\right)$ at the $t$ th stage, the optimal number of packets to transmit in state $(x, t)$ is given by

$$
k_{t}^{*}(x)=\left\{\begin{array}{c}
\max \{1 \leq k \leq \min \{x, \mathcal{B}\}: \\
\left.\triangle R_{t}(k)>\beta \cdot \triangle V_{t-1}(x-k+1)\right\} \\
\text { if } R_{t}(1)>\beta \cdot \triangle V_{t-1}(x), \\
\quad \text { otherwise. }
\end{array}\right.
$$

Moreover, it is optimal to allocate these $k_{t}^{*}(x)$ packets to the route with the lowest cost $r_{i}$.

Proof: $V_{t}(x)$ is the summation of two terms in (11). As the second term is fixed given $x$, the optimal $k_{t}^{*}$ maximizing the first term needs to be studied. Based on the definition (9), $\triangle R(\cdot)$ is decreasing in its argument. Also, $\triangle V_{t-1}(\cdot)$ is decreasing in its argument from Lemma 2. Thus, $\triangle R(k)-$ $\beta \cdot \triangle V_{t-1}(x-k+1)$ is also monotonically decreasing in $k$. Therefore, the optimal allocation at $t$ th time period with $x$ packets in queue, $k_{t}^{*}(x)$, is the largest $k$ for which this difference is positive.

Theorem 1 shows how the source node should allocate packets into different time periods. The basic idea is to progressively allocate the packets to the route with the smallest realization of $J\left(r_{(1)}\right)$ until the marginal benefit $\triangle R_{t}(i)$ drops below the marginal opportunity cost $\triangle V_{t-1}(x-i+1)$.

In order to have the optimal allocation strategies using Theorem 1, we first need to know the expected profit function $\triangle V_{t}(x), \forall t, x$. For finite number of time periods, $T$, in problem (6), the optimal dynamic programming proceeds backward using the Bellman equation [27] to obtain $\triangle V_{t}(x)$. Due to the randomness of the route number and its type, it is difficult to obtain the close-form expression of $\triangle V_{t}(x)$. Thus, we use simulation to approximate the values of $\triangle V_{t}(x)$ for different $t$ and $x$, which proceeds as follows: Start from the routing stage 0 . For each stage $t$, generate $N$ samples of the number of available routes and their types, which follow the PDF $f_{\ell}(\ell)$ and $f_{i}\left(r_{i}\right)$, respectively. For each realization and for each pair of values $(x, t)$, calculate $k_{t}^{*}(x)$ using Theorem 1. By using the conclusion of Lemma 1 , we simplify the computation of $k_{t}^{*}(x)$ and only need $O(N M)$ operations to calculate $V_{t}(x)$ for all $x$ at fixed $t$ time period. Therefore, $O(N M T)$ operations are required for the whole algorithm. Note that the computation of $V_{t}(x)$ can be done off-line, which will not increase the complexity of finding the optimal allocation for each realization.

We then study the expected profit function for infinite number of routing stages. Such scenario gives the upper-bound of the expected profit, because the source node can wait until low-cost routes being available for transmission. For infinite horizon, the maximal profit $V_{t}(x)$ in (6) can be rewritten as

$V^{*}(x)=E_{\ell, \mathbf{r}}\left[\min _{\mathbf{Q}, k}\left\{\sum_{i=1}^{\ell_{t}}\left(G(\mathcal{K})-k \cdot J\left(r_{i}\right)\right) Q_{i}(\mathbf{r})+\beta \cdot V^{*}(x-k)\right\}\right]$ or, equivalently, $V^{*}=\mathcal{T} V^{*}$, where $\mathcal{T}$ is the operator updating $V^{*}$ using (17). Assuming $S$ is the feasible set of states, The convergence proposition of the dynamic programming algorithm [27] states that: for any bounded function $V$ : $S \rightarrow \mathcal{R}$, the optimal profit function satisfies $V^{*}(x)=$ $\lim _{p \rightarrow \infty}\left(\mathcal{T}^{p} V\right)(x), \forall x \in S$. As $V(x)$ is bounded in our algorithm, we are able to apply the value iteration method to approximate the optimal $V(x)$, which proceeds as follows: Start from some initial function for $V(x)$ as $V^{0}(x)=g(x)$, where the superscript stands for the iteration number. Then, iteratively update $V(x)$ by letting $V^{p+1}(x)=\left(\mathcal{T} V^{p}\right)(x)$. The iteration process ends until $\left|V^{p+1}(x)-V^{p}(x)\right| \leq \epsilon$, for all $x$, where $\epsilon$ is the error bound for $V^{*}(x)$.

\section{Mechanism Design}

In the previous part, we have developed the optimal dynamic pricing-based routing approach. Next, our task is to find auction mechanisms that achieve the derived optimal strategy. Many auction forms can be applied to achieve the optimal strategy. Considering the truth-telling property of the secondprice auction, we focus on this mechanism in our paper. 
In a traditional second-price auction, the bidder with the highest bid wins the item and pays the second highest bid for it. In our framework, the source node is trying to find the route with the lowest cost, which implies the application of reverse second-price auction. The source node allocates the packet transmission to the route with the lowest payment bid and actually pay the second-lowest bid to the selected route. Moreover, the auction mechanism can be performed in many forms, such as open auctions and sealed-bid auctions. Open auctions allows the bidders to submit bids many times until finally only one bidder stays in the game. In sealed-bid auctions, the bidders only submit their bids once. Considering the sealed-bid auctions require less side-information and hence save the wireless resources, we analyze the sealed-bid secondprice auction for our optimal allocation policy.

It is important to note that the straightforward application of the reverse second-price auction can not guarantee the truthtelling property of the bidders. Let $\tilde{J}_{t}(r)=G\left(1, \mathcal{K}_{t+1}\right)-J(r)$ and $\tilde{r}_{t}=\tilde{J}_{t}^{-1}\left(\triangle V_{t-1}\left(x_{t}\right)\right)$, where $x_{t}$ is the packets to be transmitted from the $t$ th stage. Considering the scenario where the lowest cost of the routes $r_{(1)}^{t}>\tilde{r}_{t}$, it can be seen from Theorem 1 that no packet will be assigned for forwarding within current time period. Hence, the route with the lowest cost may have incentive to bid below their true cost and satisfy the threshold constraint. In this way, this route will win the packet and get positive payoff as the sender awards it the second lowest bid. But the expected profit of the sender will decrease according to (11). Therefore, we need to modify the second-price mechanism by using $\tilde{r_{t}}$ as the reserved price for every stage, which is the highest price that the sender agrees to pay for transmitting one packet within current time period. Specifically, given the submitted bid vector, $\mathbf{d}_{t}=\left\{d_{1, t}, d_{2, t}, \ldots, d_{\ell, t}\right\}$, the sender allocates the packet to the route with lowest bid below the reserved price and the selected route gets the payment $\max \left\{d_{(2)}, \tilde{r}\right\}$, where $d_{(2)}$ is the second lowest type of the forwarding routes.

Note that the mechanism we developed above can prevent the single route from not reporting the true cost. But in the presence of collusion of the routes, it may be not able to maintain the truth-telling property. This problem can be fixed from two aspects: First, the greediness of the selfish routes can help to prevent the collusion. Assume two routes collude to increase their profits. The collusion requires the two routes to act and share the extra gain cooperatively. But, the greediness of the routes decide that the cooperative game can not be carried out between them. The noncooperative behaviors will eventually lead to an inefficient outcome and break the collusion of the players. Second, in our scheme, the sender can discourage the collusion among the routes by setting a higher reserve price. The collusion behaviors of bidders is also referred as the bidding ring in the context of the auction theory. The optimal reserve price is analyzed in [21] to combat the collusion of bidders, which can be directly applied to our scheme for handling the route collusion.

\section{Profit Sharing among the Nodes on a Selected Route}

In the above sections, we have developed the optimal dynamic routing approach through multi-stage pricing in
MANETs and designed the mechanism of the second-price auction with reserved prices to assure the truth-telling property of each route. But, in this paper, we consider each route as an entity. Thus, the residual problem is that how to share the forwarding profits of the route defined as in (1) among the forwarding nodes on the route. Although the proposed mechanism can ensure the truth-telling of each route as one bidder, the cooperation among the nodes on one route can not be pre-assumed and truth-telling mechanisms need to be further designed for the profit-sharing problem. In this part, we will first prove that no dominant truth-telling strategy exists for each node on the selected multi-hop forwarding route in static profit-sharing scenarios. Then, the truth-telling profitsharing mechanisms are designed to enforce the cooperation among the nodes on the selected route in dynamic scenarios.

As the nodes on the same forwarding route belong to their own authorities, they will act greedily to get more profits from the total profits that the route gains, which forms a static profit-sharing game (SPSG). The players in the profitsharing game are all the nodes on the same forwarding route. The payoff of each node is defined as the profits it obtained through packet forwarding efforts, which is represented as $P_{i, j}$ for the $j$ th node on the $i$ th route. The action strategy of the $j$ th node on the $i$ th discovered route can be represented as $\left\{\alpha_{i, j}, \hat{v}_{i, j}\right\}$, where $\alpha_{i, j}$ is the the percentage of profits that this node will get for its packet forwarding efforts and $\hat{v}_{i, j}$ is the forwarding cost that it reported while performing the routebased pricing. Note that $\hat{v}_{i, j}$ may not be the true forwarding cost and our aim is to design mechanisms to enforce the truth-telling behaviors. Assume the number of hops on the $i$ th route is $h_{i}$. Let the profit-sharing vector for the $i$ th route be $\alpha_{i}=\left\{\alpha_{i, 1}, \alpha_{i, 2}, \ldots, \alpha_{i, h_{i}}\right\}$, where $\sum_{j=1}^{h_{i}} \alpha_{i, j}=1$. Denote the reported cost vector of the nodes on the $i$ th route as $\hat{\mathbf{v}}_{i}=\left\{\hat{v}_{i, 1}, \hat{v}_{i, 2}, \ldots, \hat{v}_{i, h_{i}}\right\}$. Recall that the type vector of the nodes on the $i$ th route is defined as $\mathbf{v}_{i}=\left\{v_{i, 1}, v_{i, 2}, \ldots, v_{i, h_{i}}\right\}$ and the PDF of $v_{i}$ is $\hat{f}_{i}$, which we assume to be identical for all nodes without loss of generality. Then, we study the existence of the dominant truth-telling strategies in the following theorem.

Theorem 2: There exists no dominant truth-telling strategy $\left\{\alpha_{i}, \hat{\mathbf{v}}_{\mathbf{i}}\right\}$ in the static profit-sharing game.

Proof: We prove this theorem by contradiction. Assume $\alpha_{i}^{*}$ is a dominant truth-telling profit-sharing strategy in the static profit-sharing game, which means by using $\alpha_{i}^{*}$, every forwarding node's dominant strategy on the $i$ th route is to report its true type (or cost). Equivalently, if the $j$ th node reports a higher cost, $\hat{v}_{i, j}=v_{i, j}+\epsilon$, than its true type $v_{i, j}$ while other nodes report the true value, the $j$ th node will get a lower profit. In order to show the dominant strategy $\alpha_{i}^{*}$, we need to calculate and compare the node's profit when it is cheating or not. First, the total profits of the $i$ th route are obtained and then we study the profit of each node. Based on our second-price mechanism and considering (1), the total profits of the $i$ th route can be represented as follows.

$U_{i}\left(\hat{r}_{i}\right)=\operatorname{Prob}\left(\hat{r}_{i}<r_{(1)}\left(\mathbf{r}_{-i}\right)\right) \cdot\left(E_{\mathbf{r}_{-i}}\left[r_{(1)}\left(\mathbf{r}_{-i}\right) \mid \hat{r}_{i}<r_{(1)}\left(\mathbf{r}_{-i}\right)\right]-\hat{r}_{i}\right)$,

where $\hat{r}_{i}$ is the bidding cost of the $i$ th route, which the $i$ th route believes to be the true cost, but may be not if some node on the $i$ th route is cheating by reporting a higher type value, 
and $r_{(1)}\left(\mathbf{r}_{-i}\right)$ represents the lowest cost of all routes except the $i$ th route. Without loss of generality, we assume the PDF of $r_{i}$ to be identical for all routes as $f$. By using the results of order statistics [29], we have the condition expectation of the payment as follows.

$E_{\mathbf{r}_{-i}}\left[r_{(1)}\left(\mathbf{r}_{-i}\right) \mid \hat{r}_{i}<r_{(1)}\left(\mathbf{r}_{-i}\right)\right]=\frac{1}{\left[1-F\left(\hat{r}_{i}\right)\right]^{\ell-1}} \int_{\hat{r}_{i}}^{\infty}[1-F(x)]^{\ell-1} d x$.

Noting that the probability of winning the auction for the $i$ th route is

$$
\operatorname{Prob}\left(\hat{r}_{i}<r_{(1)}\left(\mathbf{r}_{-i}\right)\right)=\left[1-F\left(\hat{r}_{i}\right)\right]^{\ell-1} .
$$

Substituting (19) and (20) into (18), the total profits can be written as

$$
U_{i}\left(\hat{r}_{i}\right)=\int_{\hat{r}_{i}}^{\infty}[1-F(x)]^{\ell-1} d x .
$$

Then, using the profit-sharing strategy $\alpha_{i}^{*}$, the profit of the $j$ th node on the $i$ th route can be calculated. We consider two cases: (a) the node reports the true type $v_{i, j}$; (b) the node cheats and reports a higher value $\hat{v}=v_{i, j}+\epsilon$. For case (a), the profit of the $j$ th node on the $i$ th route is represented as follows.

$$
\begin{aligned}
U_{i, j}\left(v_{i, j}\right) & =\alpha_{i, j}^{*} \cdot U_{i}\left(r_{i}\right) \\
& =\alpha_{i, j}^{*} \cdot \int_{r_{i}}^{\infty}[1-F(x)]^{\ell-1} d x .
\end{aligned}
$$

For case (b), the profit includes the cheating profit of reporting an extra cost $\epsilon$ and the allocated profit from the $i$ th route, which can be written as

$$
\begin{gathered}
U_{i, j}\left(\hat{v}_{i, j}\right)=\epsilon \cdot \operatorname{Prob}\left(\hat{r}_{i}<r_{(1)}\left(\mathbf{r}_{-i}\right)\right)+\alpha_{i, j}^{*} \cdot U_{i}\left(\hat{r}_{i}\right) \\
=\epsilon \cdot\left[1-F\left(r_{i}+\epsilon\right)\right]^{\ell-1}+ \\
\alpha_{i, j}^{*} \cdot \int_{r_{i}+\epsilon}^{\infty}[1-F(x)]^{\ell-1} d x .
\end{gathered}
$$

Subtracting (22) from (23), we have

$$
\begin{aligned}
& U_{i, j}\left(\hat{v}_{i, j}\right)-U_{i, j}\left(v_{i, j}\right)=\left[1-F\left(r_{i}+\epsilon\right)\right]^{\ell-1} \\
& \times\left\{\epsilon-\alpha_{i, j} \int_{r_{i}}^{r_{i}+\epsilon} \frac{[1-F(x)]^{\ell-1}}{\left[1-F\left(r_{i}+\epsilon\right)\right]^{\ell-1}} d x\right\} .
\end{aligned}
$$

From the Mean Value Theorem, we know that there exists some $\lambda \in[0,1]$ satisfying

$$
\int_{r_{i}}^{r_{i}+\epsilon} \frac{[1-F(x)]^{\ell-1}}{\left[1-F\left(r_{i}+\epsilon\right)\right]^{\ell-1}} d x=\epsilon \cdot\left(\frac{\left[1-F\left(r_{i}+\lambda \epsilon\right)\right]}{\left[1-F\left(r_{i}+\epsilon\right)\right]}\right)^{\ell-1} .
$$

And, for simplicity, let

$$
\Psi(\epsilon)=\left(\frac{\left[1-F\left(r_{i}+\lambda \epsilon\right)\right]}{\left[1-F\left(r_{i}+\epsilon\right)\right]}\right)^{\ell-1},
$$

which is a decreasing function in $\epsilon$, and has the limit

$$
\lim _{\epsilon \rightarrow 0} \Psi(\epsilon)=1 .
$$

Thus, there always exists a positive value $\delta$. When $\epsilon<\delta$, $\Psi(\epsilon)<1 / \alpha_{i, j}^{*}$. Further, by putting (25) into (24), we have

$U_{i, j}\left(\hat{v}_{i, j}\right)-U_{i, j}\left(v_{i, j}\right)=\epsilon \cdot\left[1-F\left(r_{i}+\epsilon\right)\right]^{\ell-1}\left[1-\alpha_{i, j}^{*} \cdot \Psi(\epsilon)\right]$.
Therefore, $\exists \delta$, for $\epsilon<\delta, U_{i, j}\left(\hat{v}_{i, j}\right)-U_{i, j}\left(v_{i, j}\right)>0$, which contradicts the assumption that $\alpha_{i, j}^{*}$ is a dominant truth-telling strategy. Considering such contradiction holds for any $\alpha_{i, j}^{*}$, we finally prove that there does not exist a cheat-proof strategy for the profit-sharing game.

Since there is no dominant truth-telling strategy in static profit-sharing games as Theorem 2 shows, it is necessary to design certain mechanisms to enforce the cooperation among the forwarding nodes on the same forwarding route. There are many ways to design such mechanisms. For instance, an intuitive idea is to provide over-payment to the nodes on the winning route as the compensation for their cooperative behaviors. The over-payment should be more than the cheating gain the nodes can obtain. But who is responsible for the over-payment? It is not reasonable to ask the sender for the payment-compensation. Because, in this way, the sender may have incentives to switch his/her transmission to the route with higher true cost, which asks for less over-payment. It is also a rational behavior for such route to require a less over-payment, which may make them have a positive payoff instead of losing the auction with zero payoffs. Therefore, a more practical way is to let the central-bank periodically compensate the forwarding nodes with some payments. The over-payment amount can be decided based on the VickreyClarke-Groves (VCG) mechanism [13], [21], which pays each node the difference between the routing cost without this node and the other nodes' routing cost with the presence of this node. It is important to note that the application of the VCG mechanism here does not conflict with our dynamic pricing mechanism. They are carried out separately by the central bank and the sender for ensuring the cooperation of forwarding nodes on one route and maximizing the total profits of the sender, respectively.

However, the over-payment method still requires some information of the overall topology and forward costs, which may not be available in dynamic scenarios. In order to have enforceable truth-telling mechanisms, it is reasonable to model the profit-sharing interactions as a repeated game for each route. Generally speaking, repeated games belong to the dynamic game family, which play a similar static game many times. The overall payoff in a repeated game is represented as a normalized discounted summation of the payoff at each stage game. A strategy in the repeated game is a complete plan of action, that defines the players' actions in every stage game. At the end of each stage, all the players can observe the outcome of the stage game and decide the future actions using the history of plays. The repeated profit-sharing game (RPSG) can be defined as follows.

Definition 1: Let $\Gamma$ be a static profit-sharing game and $\beta$ be a discount factor. The $T$-period profit-sharing repeated game, denoted as $\Gamma(T, \beta)$, consists of game $\Gamma$ repeated $T$ times. The repeated game payoff is given by

$$
\mathcal{P}_{i, j}=\sum_{t=0}^{T-1} \beta^{t} P_{i, j}^{t},
$$

where $P_{i, j}^{t}$ denotes the payoff of the $j$ th node on the $i$ the route in period $t$. If $T$ goes infinity, then $\Gamma(\infty, \beta)$ is referred to as the infinite repeated game. 
Note that Nash Equilibrium [10], [11] is an important concept to measure the outcome of the SPSG, which is a set of strategies, one for each player, such that no selfish player has incentive to unilaterally change his/her action. However, the selfishness of players will result in inefficient non-cooperative Nash Equilibriums in static games. As for dynamic games, Subgame Perfect Equilibrium (SPE) can be used to study the game outcomes, which is an equilibrium such that users' strategies constitute a Nash equilibrium in every subgame [10], [11] of the original game. In the RPSG, since the game is not played only once, the players is able to make decisions conditioning on past moves for better outcomes, thus allowing for reputation effects and retribution. Therefore, in order to measure the outcome of the RPSG, we apply the Folk Theorems [10], [30] of the infinite repeated games to have the following theorem.

Theorem 3: In RPSG, there exists a discount factor $\hat{\beta}<1$ such that any feasible and individually rational payoff can be enforced by an equilibrium for any discount factor $\beta \in(\hat{\beta}, 1)$.

The above theorem illustrates that feasible profit-sharing outcomes can be enforced in the RPSG when no dominant strategy is available. However, it didn't answer the question that how the feasible profit-sharing outcomes can be enforced, that is, how to design the enforcing mechanisms in the RPSG. First, we define two strategies: the cooperative strategy and non-cooperative strategy. In cooperative strategy, the node will report the true forwarding cost; in non-cooperative strategy, the node will report a very high forwarding cost so that the route with this node will not be selected for packet forwarding. Similar to [30]-[32], we propose the following mechanism to enforce truth-telling strategies for the RPSG.

\section{CArtel Maintenance Profit-sharing (CAMP) mecha-} nism:

(1) Each node on the selected route plays the cooperative strategy at the first stage;

(2) If the cooperation strategy is played in stage $t$ and $U_{i}=$ $\sum_{j=1}^{h_{i}} P_{i, j} \geq \tilde{U}$, each node plays the cooperative strategy in stage $t+1$;

(3) If the cooperation strategy is played in stage $t$ and $U_{i}<$ $\tilde{U}$, each node switches to a punishment phase for $\mathcal{T}-1$ stages, in which the non-cooperative strategy is played regardless of the realized outcomes. At the $\mathcal{T}$ th period, each node switches back to the cooperative strategy.

Note that $\tilde{U}$ is the cartel maintenance threshold. Similar to [30]-[32], the optimal $\tilde{U}$ and $\mathcal{T}$ can be obtained using the routing statistics. The proposed CAMP mechanism uses the non-cooperative punishment launched by all nodes to prevent any deviating strategies from the cooperative strategy. Specifically, although the deviating behaviors may benefit a node at current stage, its payoff will be decreased more in future stages. By using the CAMP mechanism, the truth-telling profit sharing is enforceable among the nodes on the selected route. Based on Theorem 3, we can enforce any feasible profit sharing strategy such as equal sharing or proportional sharing according to the effort of each node.

\section{Simulation Studies}

In this section, we evaluate the performance of the proposed dynamic pricing approach in multi-hop ad hoc networks. We
TABLE I: Simulation Parameters

\begin{tabular}{||l|l||}
\hline \hline Node Density & $10,20,30$ \\
Minimum Velocity $\left(v_{\min }\right)$ & $10 \mathrm{~m} / \mathrm{s}$ \\
Maximum Velocity $\left(v_{\max }\right)$ & $30 \mathrm{~m} / \mathrm{s}$ \\
Average Pause time & $100 \mathrm{~seconds}$ \\
Dimensions of Space & $1000 \mathrm{~m} \times 1000 \mathrm{~m}$ \\
Maximum Transmission Range & $100 \mathrm{~m}$ \\
Average Packet Inter-Arrival Time & $1 \mathrm{~seconds}$ \\
Data Packet Size & 1024 bytes \\
Link Bandwidth & $8 \mathrm{Mbps}$ \\
\hline \hline
\end{tabular}

consider an ad hoc network where $\mathcal{N}$ nodes are randomly deployed inside a rectangular region of $10 \gamma \mathrm{m} \times 10 \gamma \mathrm{m}$ according to the 2-dimension uniform distribution with the maximal transmission range $\gamma=100 \mathrm{~m}$ for each node. Let $\lambda=\mathcal{N} \pi / 100$ denote the normalized node density, that is, the average number of neighbors for each node in the network. Each node moves according to the random waypoint model [33]: a node starts at a random position, waits for a duration called the pause time, then randomly chooses a new location and moves toward the new location with a velocity uniformly chosen between $v_{\min }$ and $v_{\max }$. When it arrives at the new location, it waits for another random pause time and repeats the process. The physical layer assumes that two nodes can directly communicate with each other successfully only if they are in each other's transmission range. The MAC layer protocol simulates the IEEE 802.11 Distributed Coordination Function (DCF) with a four-way handshaking mechanism [34]. Table I shows all simulation parameters. Note that each source-destination pair is formed by randomly picking two nodes in the network. And, multiple routes with different hop number may exist for each sourcedestination pair. Since the routes with the least hop number have much higher probabilities to achieve lower costs, without loss of generality, we only consider the least-hop routes as the bidding routes for simplicity in the proposed optimal dynamic auction framework. Considering the mobility of each node, its forwarding cost is no longer a fixed value and we assume that its $\operatorname{PDF} \hat{f}(v)$ follows the uniform distribution $\mathcal{U}[\bar{u}, \underline{u}]$, which has the mean $\mu$ and the variance $\sigma^{2}$. Thus, using the Central Limit Theorem [29], the cost of a $h$-hop route can be approximated by the normal distribution with the mean $h \cdot \mu$ and variance $h \cdot \sigma^{2}$. In our simulation, we first study the dynamics of MANET and then illustrate the performance of our proposed framework for different network settings.

In order to study the dynamics of MANET, we first conduct simulations to study the hop number on the least-hop route for source-destination pairs. Let $\hbar\left(n_{i}, n_{j}\right)=\left\lceil\operatorname{dist}\left(n_{i}, n_{j}\right) / \gamma\right\rceil$ denote the minimum number of hops needed to traverse from node $i$ to node $j$, where $\operatorname{dist}\left(n_{i}, n_{j}\right)$ denotes the physical distance between node $i$ and $j$, and let $\tilde{\hbar}\left(n_{i}, n_{j}\right)$ denote the number of hops on the actual least-hop route between the two nodes. Note that we simulate $10^{6}$ samples of topologies to study the dynamics of MANET. Firstly, Figure 3 shows the approximated cumulative probability mass function (CMF) of the difference between the $\tilde{\hbar}\left(n_{i}, n_{j}\right)$ and $\hbar\left(n_{i}, n_{j}\right)$ for different node densities. Based on these results, the average number of hops associated to the least-hop route from node $i$ to $j$ can be approximated using the $\operatorname{dist}\left(n_{i}, n_{j}\right), \gamma$, and the 


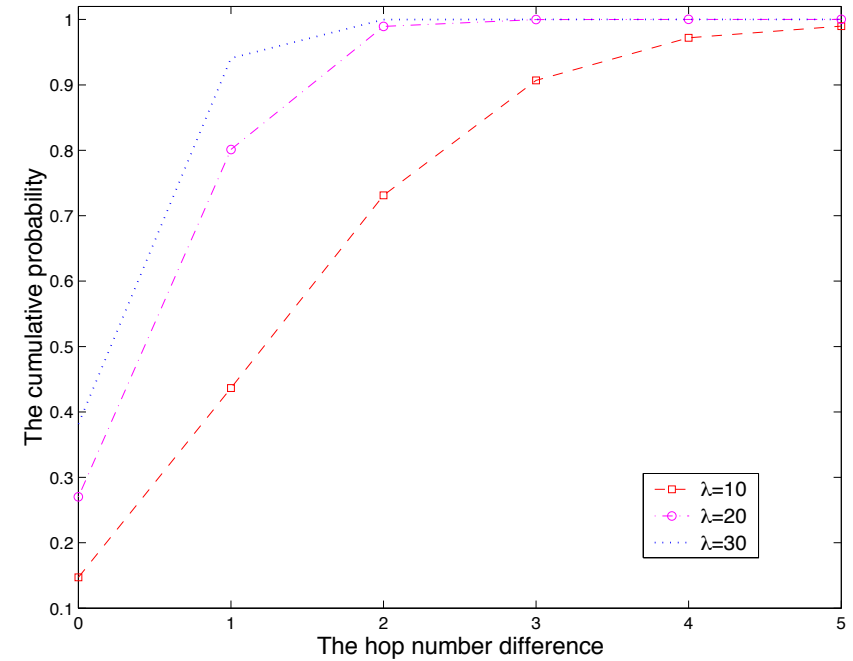

Fig. 3: The cumulative probability mass function of the hopnumber difference between the $h(u, v)$ and $r(u, v)$.

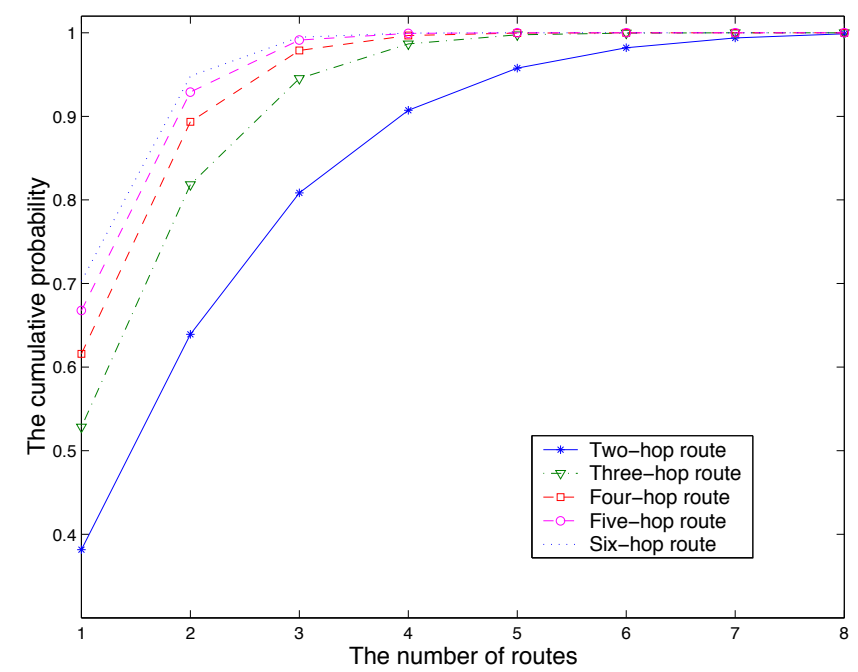

Fig. 4: The cumulative probability mass function of the number of the least-hop route when the node density is 10 .

corresponding CMF of hop difference. Also, it can be seen from Figure 3 that lower node density results in having a larger number of hops for the least-hop routes, since the neighbor nodes are limited for packet forwarding in such situations. Secondly, we study the time and path diversity of MANET by finding the maximum number of least-hop routes for the source-destination pair. Note that there may exist the scenarios where the node may be on multiple least-hop forwarding routes for the same source-destination pair. For simplicity, we assume during the route discovery phase, the destination randomly picks one of such routes as the routing candidates and feedbacks the routing information of node-disjoint leasthop routes to the source. Figure 4 shows the CMF of the number of the least-hop routes for different hop number when the node density is 10 . The results for the node density 20 and 30 are shown in Figure 5 and Figure 6, respectively. It can be seen from the above figures that when the node density is increasing, the probability of having more routes between each source-destination pair is becoming much higher. Such facts

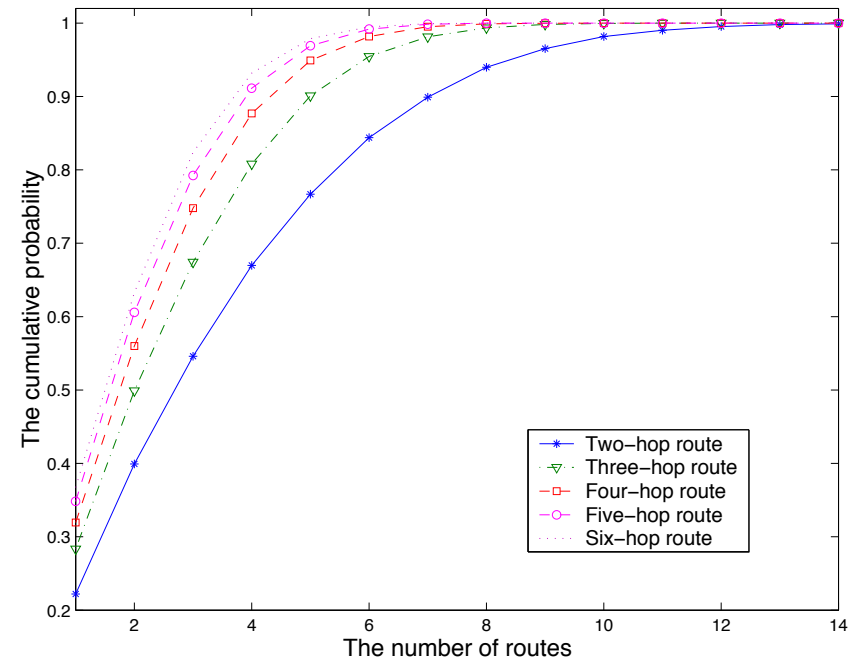

Fig. 5: The cumulative probability mass function of the number of the least-hop route when the node density is 20 .

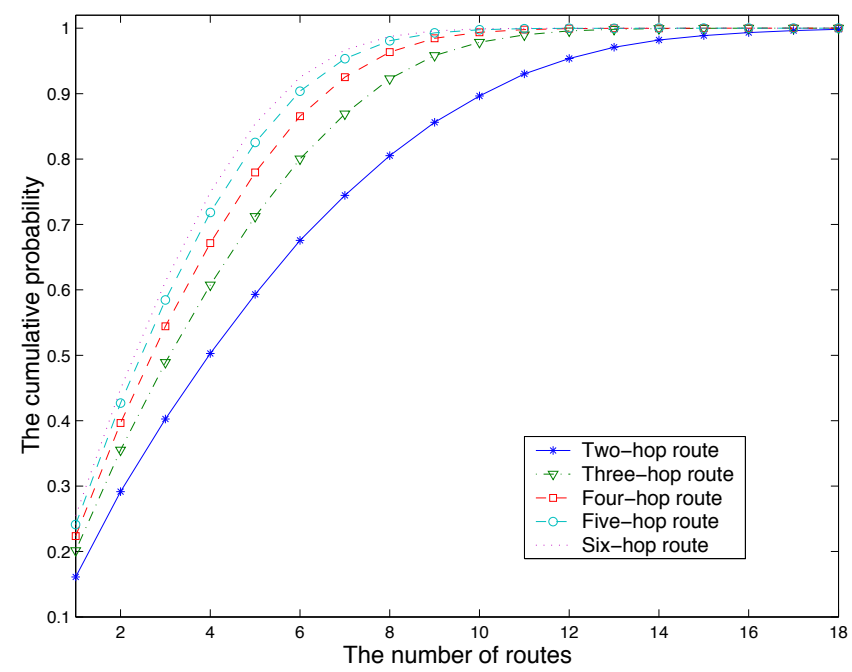

Fig. 6: The cumulative probability mass function of the number of the least-hop route when the node density is 30 .

also indicate a higher order of path diversity can be exploited when each node has more neighbors. Moreover, the possibility of getting more routes for the route with more hops is much lower since the path diversity for multi-hop routing is limited by the forwarding node with the worst neighboring situation. Therefore, the number of routing candidates and their types can be approximated using the above results.

In the following parts, we consider the performance for three different schemes: our scheme with finite time horizon, our scheme with infinite time horizon and the fixed allocation scheme. Note that the infinite time horizon can not be achieved in real application. But it can serve as a upper bound for measuring the performance of our scheme. The fixed scheme allocates a fixed number of packets into each stage while also using the optimal auction at each stage. Assume the cheat-proof profit sharing mechanisms are in place to ensure the cooperation of the forwarding nodes on the same route. Let the benefit function be $G(\mathcal{K})=g \cdot k$, where $g$ is the benefit of successfully transmitting one packet. Note that the 


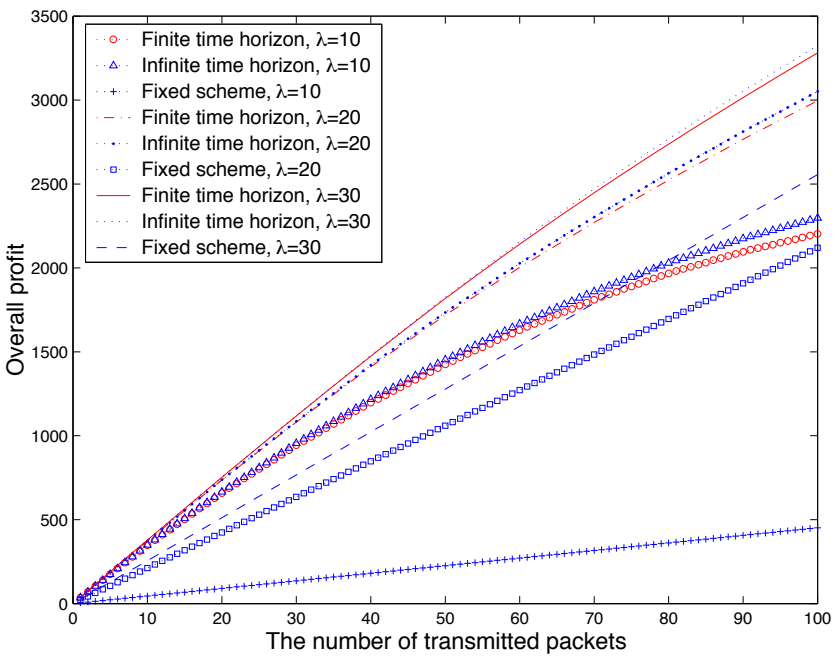

Fig. 7: The overall profits of our scheme with finite time horizon, our scheme with infinite time horizon and the fixed scheme.

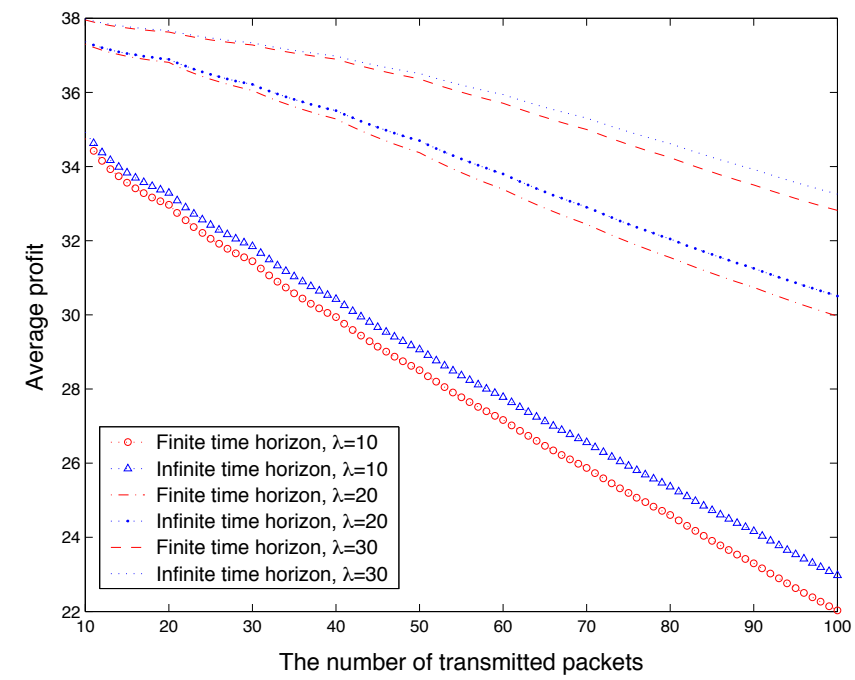

Fig. 8: The average profits of our scheme with finite time horizon, our scheme with infinite time horizon and the fixed scheme.

simulation parameters are set as $T=20, M=100$ and $\mathcal{B}=10$. Let $g=60, \bar{u}=10$, and $\underline{u}=15$. In Figure 7 , we compare the overall profits of the three schemes for different node densities. The concavity of the simulated value functions of our scheme matches the theoretical statement in Lemma 2. It can be seen from the figure that our scheme achieves significant performance gains over the fixed scheme, which mainly comes from the time diversity exploited by the dynamic approach. We observe that the performance gap of the two schemes becomes larger when the node density decreases. Thus, in order to increase the profits under the situations of low node densities, it becomes much more important to exploit the time diversity. Also, the total profits of our scheme increases with the increment of the node density due to the higher order of path diversity. Besides, since the performance gap between the schemes with finite and infinite time horizon is small, only a few routing stages are required to exploit the time diversity.

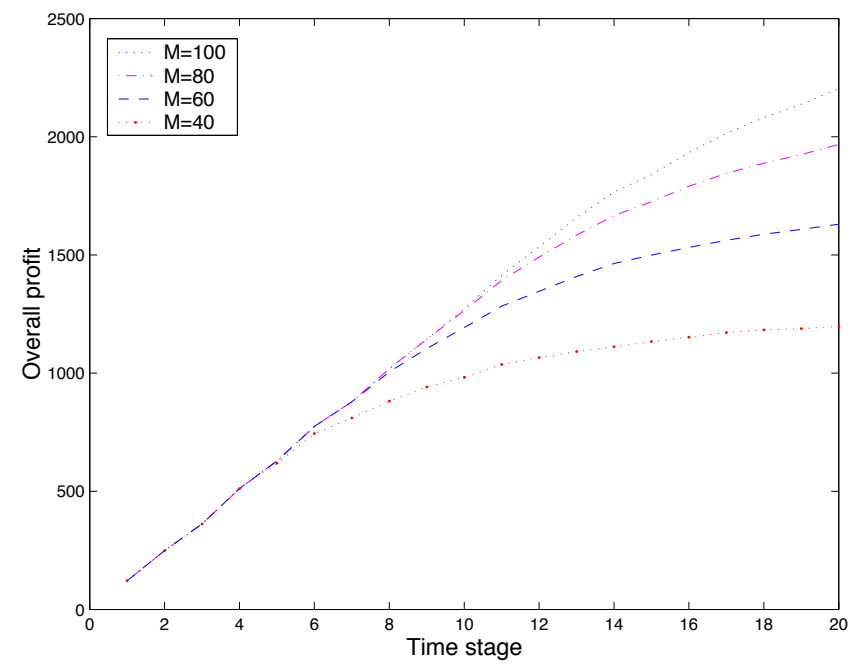

Fig. 9: The overall profits of our scheme with different packets to be transmitted when the node density is 10 .

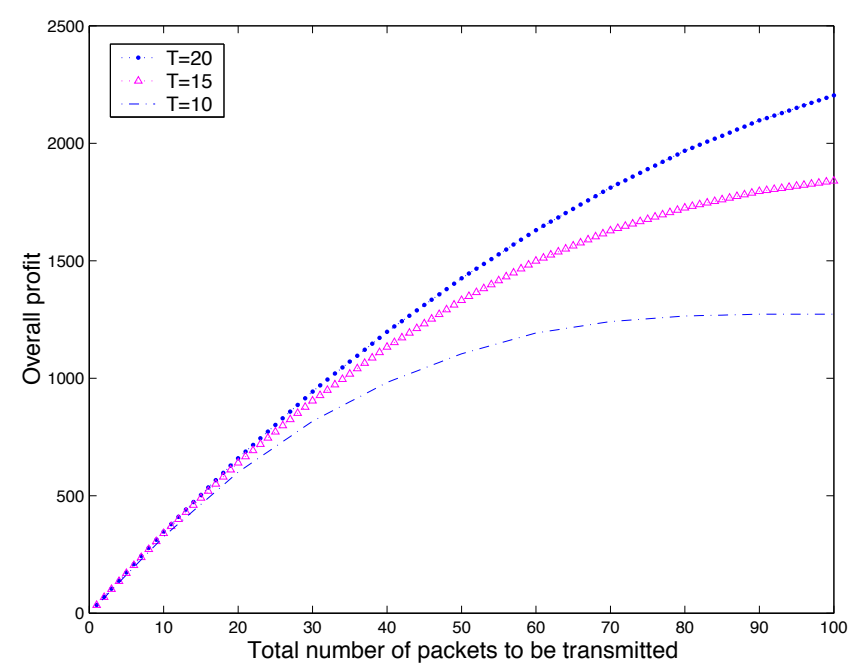

Fig. 10: The overall profits of our scheme with different time stages when the node density is 10 .

In Figure 8, the average profits of the three schemes are shown for different node densities. This figure shows that the average profit of transmitting one packet decreases as there are more packets to be transmitted. It is because the packets need to share the limited routing resources from both the time diversity and path diversity. When the node density is 30 , the average profit degrades much slower than other cases since the potential of utilizing both the time diversity and path diversity is high. The overall profits of our scheme with finite time horizon are compared for different total packets in Figure 9 for node density being 10 . This figure shows that the overall profits increases with more routing stages due to the time diversity. Also, the saturation behavior can be observed when using more stages. In Figure 10, the overall profits are compared for different time stages. Considering the limited routing resource, the overall profits saturate when the packet number is high. 


\section{CONCLUSIONS}

In this paper, we study how to conduct efficient pricingbased routing in self-organized MANET by assuming that the packet-forwarding will incur a cost to the relay node and the successful transmission brings benefits to the sourcedestination pairs. Considering the dynamic nature of MANET, we model the routing procedure as a multi-stage pricing game and propose an optimal dynamic pricing-based routing approach to maximize the payoffs of the source-destination pair while keeping the forwarding incentives of the relay nodes on the selected routes by optimally pricing their packetforwarding services through the auction protocol. It is important to notice that not only the path diversity but also the time diversity in MANETs can be exploited by our dynamic pricing-based approach. Also, the optimal dynamic auction algorithm is developed to achieve the optimal allocation of packets to be transmitted, which provides the corresponding pricing rules while taking into consideration of the node's mobility and the routing dynamics. Extensive simulations have been conducted to study the performances of the proposed approach. The results illustrate that the proposed approach achieves significant performance gains over the existing static routing approaches.

\section{APPENDIX}

\section{Proof of Lemma 2}

Proof: First, we prove that $\triangle V_{t}(x)$ is decreasing in $x$ at any fixed time period $t$. Note that the induction method is used to prove this part of Lemma 2. For $t=0$, the lemma obviously holds since $V_{0}(x)=0$ for all $x$. Assume the inductive hypothesis for period $t-1$ as $\Delta V_{t-1}(x) \geq \triangle V_{t-1}(x+1)$. Then, we will show that if the inductive hypothesis holds, $\triangle V_{t}(x)$ also decreases.

Consider a realization of $\ell_{t}$ routes and their cost vector $\mathbf{r}=\left(r_{1}, r_{2}, \ldots, r_{\ell_{t}}\right)$. Define the inner maximized term in (10) as follows

$$
U_{t}\left(x, \ell_{t}, \mathbf{r}\right)=\max _{0 \leq k \leq \min \{\mathcal{B}, x\}}\left\{R_{t}(k)+\beta \cdot V_{t-1}(x-k)\right\},
$$

and define the difference function as

$$
\triangle U_{t}\left(x, \ell_{t}, \mathbf{r}\right)=U_{t}\left(x, \ell_{t}, \mathbf{r}\right)-U_{t}\left(x-1, \ell_{t}, \mathbf{r}\right) .
$$

Thus $\triangle V_{t}(x)$ can be obtained as

$$
\triangle V_{t}(x)=E_{\ell_{t}, \mathbf{r}}\left[\triangle U_{t}\left(x, \ell_{t}, \mathbf{r}\right)\right] .
$$

For simplicity and without loss of generality, we omit the arguments $\ell_{t}, \mathbf{r}$ in $\triangle U_{t}\left(x, \ell_{t}, \mathbf{r}\right)$ and simply use $\triangle U_{t}(x)$. Moreover, it can be seen from (32) that it is sufficient to prove that $\triangle U_{t}(x)$ is decreasing in $x$ for the proof that $\triangle V_{t}(x)$ is decreasing in $x$.

Using the inductive hypothesis and Lemma 1, we have the constraint on $k_{t}^{*}(x+1)$ as

$$
k_{t}^{*}(x) \leq k_{t}^{*}(x+1) \leq k_{t}^{*}(x)+1 .
$$

Based on the constraint, we then study the value of $\triangle U_{t}(x+$ 1) for the two possible outcomes, $k_{t}^{*}(x+1)=k_{t}^{*}(x)$ and $k_{t}^{*}(x+1)=k_{t}^{*}(x)+1$ :
1). If $k_{t}^{*}(x+1)=k_{t}^{*}(x)$, then $\triangle U_{t}(x+1)=\beta$. $\triangle V_{t-1}\left(x-k_{t}^{*}(x)+1\right)$ from (30) and (31). Also, from the optimal condition of $k$ in (12), we know

$\triangle R_{t}\left(k_{t}^{*}(x+1)+1\right) \leq \beta \cdot \triangle V_{t-1}\left(x+1-\left(k_{t}^{*}(x+1)+1\right)+1\right)$.

Considering $k_{t}^{*}(x+1)=k_{t}^{*}(x)$, (34) can be rewritten as

$$
\triangle R_{t}\left(k_{t}^{*}(x)+1\right) \leq \beta \cdot \triangle V_{t-1}\left(x-k_{t}^{*}(x)+1\right) .
$$

2). Similarly, If $k_{t}^{*}(x+1)=k_{t}^{*}(x)+1$, then $\triangle U_{t}(x+1)=$ $\triangle R_{t}\left(k_{t}^{*}(x)+1\right)$ from (30) and (31), and

$$
\triangle R_{t}\left(k_{t}^{*}(x)+1\right)>\beta \cdot \triangle V_{t-1}\left(x-k_{t}^{*}(x)+1\right) .
$$

Thus, it can be concluded from the above two cases that $\triangle U_{t}(x+1)$ satisfies

$\triangle U_{t}(x+1)=\max \left\{\triangle R_{t}\left(k_{t}^{*}(x)+1\right), \beta \cdot \triangle V_{t-1}\left(x-k_{t}^{*}(x)+1\right)\right\}$.

Consider now $\triangle U_{t}(x+1)$ and $\triangle U_{t}(x)$ and compare their values. Given the constraint on $k_{t}^{*}(x)$ by Lemma 1 , the value of $\triangle U_{t}(x+1)$ in (37), and considering that $\triangle R_{t}(m)$ and $\triangle V_{t-1}(m)$ decrease in their arguments, we have the following expressions.

$$
\begin{aligned}
& \Delta U_{t}(x) \\
= & \max \left\{\triangle R_{t}\left(k_{t}^{*}(x-1)+1\right),\right. \\
& \left.\beta \cdot \triangle V_{t-1}\left(x-1-k_{t}^{*}(x-1)+1\right)\right\} \\
\geq & \max \left\{\triangle R_{t}\left(k_{t}^{*}(x)+1\right), \beta \cdot \triangle V_{t-1}\left(x-\left(k_{t}^{*}(x)-1\right)\right)\right\} \\
= & \triangle U_{t}(x+1) .
\end{aligned}
$$

Therefore, the first part of Lemma 2 is proved by the above discussion.

Next, we show that $\triangle V_{t}(x)$ is increasing in $t$ for any fixed $x$. Similarly, it suffices to prove the statement for a particular realization $\ell_{t}, \mathbf{r}$. Following the results in (37), we get that

$$
\triangle U_{t}(x) \geq \beta \cdot \triangle V_{t-1}\left(x-k_{t}^{*}(x)\right),
$$

and from the fact that $\triangle V_{t-1}(\cdot)$ is decreasing, we have

$$
\triangle U_{t}(x) \geq \beta \cdot \triangle V_{t-1}(x) .
$$

As taking the expectation with respect to $\ell_{t}, \mathbf{r}$ on both sides of (40) does affect the inequality, we prove

$$
\triangle V_{t}(x) \geq \triangle V_{t-1}(x) \text {. }
$$

\section{REFERENCES}

[1] C. Perkins, Ad Hoc Networking, Addison-Wesley, 2000

[2] C. K. Toh, Ad Hoc Mobile Wireless Networks: Protocols and Systems, Prentice Hall PTR, 2001.

[3] S. Marti, T. J. Giuli, K. Lai, and M. Baker, "Mitigating Routing Misbehavior in Mobile Ad Hoc Networks," in ACM MobiCom 2000, Aug. 2000.

[4] P. Michiardi and R. Molva, "Core: a COllaborative REputation Mechanism to Enforce Node Cooperation in Mobile Ad Hoc Networks," in IFIP - Communications and Multimedia Security Conference, 2002.

[5] S. Buchegger and J.-Y. Le Boudec, "Performance Analysis of the CONFIDANT Protocol," in ACM MobiHoc, 2002.

[6] S. Zhong, J. Chen, and Y. R. Yang, "Sprite: A Simple, CheatProof, Credit-Based System for Mobile Ad-Hoc Networks," in IEEE INFOCOM, 2003. 
[7] W. Yu and K. J. R. Liu, "Attack-Resistant Cooperation Stimulation in Autonomous Ad Hoc Networks," IEEE J. Select. Areas Commun.: Autonomic Communication Systems, vol. 23, pp. 2260-2271, Dec. 2005.

[8] Z. Ji, W. Yu, and K. J. R. Liu, "An optimal dynamic pricing framework for autonomous mobile ad hoc networks," in Proc. IEEE INFOCOM'06, Barcelona, 2006.

[9] V. Srinivasan, P. Nuggehalli, C. F. Chiasserini, and R. R. Rao, "Cooperation in Wireless Ad Hoc Networks," in IEEE INFOCOM'03, 2003.

[10] D. Fudenberg and J. Tirole, Game Theory, The MIT Press, Cambridge, Massachusetts, 1991.

[11] M. J. Osborne and A. Rubinstein, A Course in Game Theory, The MIT Press, Cambridge, Massachusetts, 1994.

[12] Z. Ji, W. Yu, and K. J. R. Liu, "Cooperation Enforcement in Autonomous MANETs under Noise and Imperfect Observation," in Proc. IEEE SECON'06, 2006.

[13] L. Anderegg and S. Eidenbenz, "Ad hoc-VCG: A truthful and costefficient routing protocol for mobile ad hoc networks with selfish agents," in ACM MobiCom'03, 2003, pp. 245-259.

[14] S. Eidenbenz, G. Resta, and P. Santi, "COMMIT: a sender-centric truthful and energy-efficient routing protocol for ad hoc networks with selfish nodes," in IEEE Parallel and Distributed Processing Symposium, 2005.

[15] S. Zhong, L. Li, Y. Liu, and Y. R. Yang, "On designing incentivecompatible routing and forwarding protocols in wireless ad-hoc networks - an integrated approach using game theoretical and cryptographic techniques," ACM MobiCom'05, 2005.

[16] J. Feigenbaum, C. Papadimitriou, R. Sami, and S. Shenker, "A BGPbased mechnism for lowest-cost routing," in the 21st Symposium on Principles of Distributed Computing, 2002, pp. 173-182.

[17] N. Nisan and A. Ronen, "Computationlly feasible VCG mechnisms," in $A C M E C^{\prime} 00,2000$, pp. 242-252.

[18] K. Chen and K. Nahrstedt, "iPass: an incentive compatible auction scheme to enable packet forwarding service in MANET," in ICDCS'04, 2004.

[19] H. Frey, J. K. Lehnert and P. Sturm, "UbiBay: an auction system for mobile multihop ad-hoc networks," in ICEIS'04, 2004.

[20] W. Wang, X. Li, and Y. Wang, "Truthful multicast routing in selfish wireless networks," in ACM MobiCom'04, Sep. 2004.

[21] V. Krishna, Auction Theory, Academic Press, 2002.

[22] J. Broch, D. A. Maltz, D. B. Johnson, Y. Hu, and J. G. Jetcheva, "A performance comparison of multi-hop wireless ad hoc network routing protocols," in ACM MobiCom'98, 1998.

[23] P. Johansson, T. Larsson, N. Hedman, B. Mielczarek, and M. Degermark, "Scenario-based performance analysis of routing protocols for mobile ad-hoc networks," in ACM MobiCom'99, August 1999.

[24] C. E. Perkins and E. M. Royer, "Ad-hoc on-demand distance vector routing," in Second IEEE Workshop on Mobile Computing Systems and Applications (WMCSA99), February 1999.

[25] T. M. Cover and J. A. Thomas, Information Theory, John Wiley \& Sons, Second edition, 1991.

[26] R. Myerson, "Optimal auction design," Math. Oper. Res., vol. 6, 1981.

[27] D. Bertsekas, Dynamic Programming and Optimal Control, vol. 1,2, Athena Scientific, Belmont, MA, Second edition, 2001.

[28] G. Vulcano, G. V. Ryzin, and C. Maglaras, "Optimal dynamic auctions for revenue management," Management Science, 48(11).

[29] A. Papoulis and S. U. Pillai, Probability, Random Variables and Stochastic Process, McGraw Hill, Fourth edition, 2002.

[30] D. Abreu, P. Milgrom, and D. Pearce, "Toward a theory of discounted repeated games with imperfect monitoring," Econometrica, 1990.

[31] R. H. Porter, "Optimal Cartel Trigger Price Strategies," Journal of Economic Theory, April 1983.

[32] Z. Han, Z. Ji and K. J. Ray Liu, "A cartel maintenance framework to enforce cooperation in wireless networks with selfish users," to appear in IEEE Trans. Wireless Commun., 2008.

[33] D. B. Johnson and D. A. Maltz, "Dynamic Source Routing in Ad Hoc Wireless Networks, Mobile Computing," In Mobile Computing, edited by Tomasz Imielinski and Hank Korth, chapter 5, pages 153-181, Kluwer Academic Publishers, 1996.
[34] IEEE Computer Society LAN MAN Standards Committee, "Wireless LAN Medium Access Control (MAC) and Physical Layer (PHY) Specifications, IEEE Std 802.11-1007,” The Institue of Electrical and Electrics Engineers.

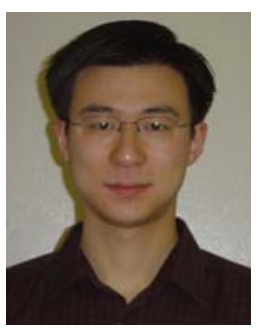

Zhu Ji received a Ph.D. degree in Electrical and Computer Engineering from University of Maryland, College Park in May 2007. He received B.S. and M.S. degrees in Electronic Engineering from Tsinghua University, Beijing, China, in 2000 and 2003, respectively.

He is currently with Qualcomm, San Diego, CA. From 2003 to 2007, he was a graduate research assistant in the Communication and Signal Processing Laboratory, University of Maryland, College Park. From 2000 to 2002, he was a visiting student (research intern) in the Wireless and Networking Group at Microsoft Research Asia, Beijing, China. His research interests are in wireless communications and networking.

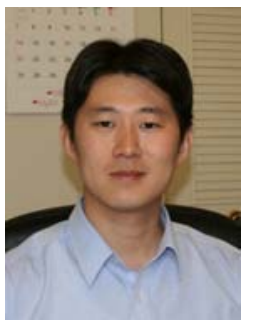

Wei Yu received the B.S. degree in computer science from the University of Science and Technology of China (USTC), Hefei, China, in 2000, the M.S. degree in computer science from Washington University, St. Louis, MO, in 2002, and the Ph.D. degree in electrical engineering from the University of Maryland, College Park, in 2006.

From 2000 to 2002, he was a Graduate Research Assistant at Washington University. From 2002 to 2006, he was a Graduate Research Assistant with the Communications and Signal Processing Laboratory and the Institute for Systems Research, University of Maryland. He joined Microsoft Corporation, Redmod, WA, in 2006. His research interests include network security, wireless communications and networking, game theory, wireless multimedia, handwriting recognition, and pattern recognition.

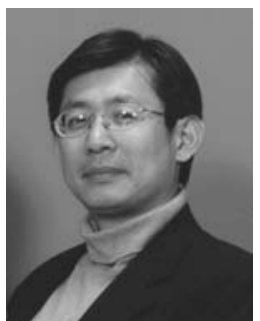

K. J. Ray Liu (F03) received the B.S. degree from the National Taiwan University and the Ph.D. degree from UCLA, both in electrical engineering. He is Professor and Associate Chair, Graduate Studies and Research, of Electrical and Computer Engineering Department, University of Maryland, College Park. His research contributions encompass broad aspects of wireless communications and networking, information forensics and security, multimedia communications and signal processing, bioinformatics and biomedical imaging, and signal processing algorithms and architectures.

Dr. Liu is the recipient of numerous honors and awards including best paper awards from IEEE Signal Processing Society (twice), IEEE Vehicular Technology Society, and EURASIP; IEEE Signal Processing Society Distinguished Lecturer, EURASIP Meritorious Service Award, and National Science Foundation Young Investigator Award. He also received various teaching and research recognitions from University of Maryland including university-level Distinguished Scholar-Teacher Award, Invention of the Year Award, Fellow of Academy for Excellence in Teaching and Learning, and college-level Poole and Kent Company Senior Faculty Teaching Award.

Dr. Liu is Vice President Publications and on the Board of Governor of IEEE Signal Processing Society. He was the Editor-in-Chief of IEEE Signal Processing Magazine and the founding Editor-in-Chief of EURASIP Journal on Applied Signal Processing. 